\title{
Appreciation to Our Peer Reviewers in 2019
}

\author{
Jae-Young Lim \\ Editor-in-Chief of Annals of Geriatric Medicine and Research, Seoul National University Bundang Hospital, Seongnam Korea
}

As we begin the first issue of 2020, the Editorial Board, Associate Editors, and Editor-in-Chief of Annals of Geriatric Medicine and Research (AGMR) would like to thank our reviewers for their ongoing service and commitment to AGMR. We rely on the clinical and research expertise of peer reviewers to ensure that the manuscripts submitted to the journal undergo a thorough, fair, and timely review.

Over the last year, AGMR has continued to move forward as a growing platform for the academic needs of geriatrics and gerontology professionals and researchers. In November 2019, AGMR was accepted for inclusion in Scopus, an abstract and citation database from Elsevier. As a fast-growing journal in the multidisciplinary aging research field, our success in entering the scholarly universe of Scopus will improve the visibility of our scientific literature to researchers working in relevant fields. This achievement would not have been possible without the voluntary contributions of our reviewers to improve the scientific quality of our journal.

AGMR invited 62 experts to peer review manuscripts in 2019, some of whom received multiple invitations. With deep gratitude, I would like to particularly acknowledge the dedication of two of these peer reviewers, Drs. Jongkyoung Choi and Sun-Wook Kim, who were selected to receive Best Reviewer awards. Once again, we appreciate the rigorous and conscientious efforts of all of our reviewers and humbly request their ongoing interest and support in 2020 .

\section{Names of AGMR reviewers in 2019}

Jae Won Beom

Chung-Ang University Hospital

Han Sung Choi

Kyunghee University Hospital

Jung Yeon Choi

Seoul National University Bundang Hospital

Eun-Jung Han

National Health Insurance Corporation

Il-Young Jang

Asan Medical Center, Seoul

Chang Wook Jeong

Seoul National University Hospital

Chang-O Kim

The Visiting Doctors Program of Medical Home, Korea

Gunn Hee Kim

National Medical Center

Jung Hee Kim

Seoul National University Hospital
Sung Tae Cho

Hallym University Hospital

Jae Kyung Choi

Konkuk University Hospital

Ming-Yueh Chou

Kaohsiung Veterans General Hospital, Taiwan

Ji Won Han

Seoul National University Bundang Hospital

Soong-Nang Jang

Chung-Ang University Hospital

Hee Won Jung

Seoul National University Hospital

Dae Yul Kim

Asan Medical Center, Seoul

Il-Young Kim

Gachon University Gil Hospital

Kyoung-Min Kim

Seoul National University Bundang Hospital
Eun Joo Choi

Seoul National University Bundang Hospital

Jong Kyoung Choi

National Medical Center

Hyuk Ga

Incheon Eun-Hye Hospital

Hwan Sik Hwang

Hanyang University Hospital

Sung Man Jang

Kyungpook National University Hospital

Bu Kyung Kim

Kosin University Hospital

Do Hyun Kim

Dankook University Hospital

Ju yeon Kim

The University of Seoul

Kwang-Il Kim

Seoul National University Bundang Hospital 


\author{
Sun-Wook Kim \\ Seoul National University Hospital \\ Won Kim \\ Asan Medical Center, Seoul \\ Taro Kojima \\ The University of Tokyo, Japan \\ Ho Jun Lee \\ Dongguk University Ilsan Hospital \\ Jong Rok Lee \\ Gachon University Gil Hospital \\ Sang Ah Lee \\ Jeju University Hospital \\ Seok Bum Lee \\ Dankook University Hospital \\ Byung-Mo Oh \\ Seoul National University Hospital \\ Ji Hong Park \\ Seoul National University Bundang Hospital \\ Ki Young Son \\ Asan Medical Center \\ Jae Myoung Suh \\ KAIST \\ Jong Lull Yoon \\ Hallym University Hospital
}

Sun Young Kim

Kyunghee University Hospital

Won-Seok Kim

Seoul National University Bundang Hospital

Eun Joo Lee

Asan Medical Center, Seoul

In-Sik Lee

Konkuk University Hospital

Jong-Seok Lee

Kyunghee University Hospital

Sang Yoon Lee

Chung Ang University Hospital

Jae-Young Lim

Seoul National University Bundang Hospital

Seung-Lyul Oh

Seoul National University Bundang Hospital

Myong Hwa Park

Chungnam National University Hospital

Hee Jin Song

Inha University Hospital

Ye Won Suh

Seoul National University Bundang Hospital

Sol Ji Yoon

Kangwon University Hospital
Mi Ji Kim

Kyoung Hee University Hospital

Young Sang Kim

CHA University Hospital

Han Cheol Lee

Pusan National University Hospital

Ji Eun Lee

CHA University Hospital

Jung Jae Lee

Dankook University Hospital

Seung Yeol Lee

Soonchunhyang University Bucheon Hospital

Yong Su Lim

Gachon University Gil Hospital

In Cheol Park

Yonsei University Severance Hospital

Kyoung-Ho Seo

Hallym University Hospital

Kee-Ho Song

Konkuk University Medical Graduate School

Jun-Il Yoo

Gyeongsang National University Hospital

Corresponding Author: Jae-Young Lim, MD, PhD

Department of Rehabilitation Medicine, Seoul National University Bundang

Hospital, Seoul National University College of Medicine, 82 Gumi-ro,

173beon-gil, Bundang-gu, Seongnam 13620, Korea

E-mail: drlim1@snu.ac.kr

ORCID: https://orcid.org/0000-0002-9454-0344

Received: March 20, 2020; Accepted: March 21, 2020 